\title{
Inhibitory Potential of Molecular Mechanism of Pathogenesis with Special Reference to Biofilm Inhibition by Chemogenic Zinc Oxide Nanoparticles
}

\author{
S. Karthick Raja Namasivayam ${ }^{1, *}$, D. Shyamsundar ${ }^{1}$, M.M. Prabanch ${ }^{1}$, R.S. Arvind Bharani ${ }^{1}$, \\ G.P. Avinash 1
}

1 Centre for Bioresource Research.\& Development (C-BIRD), Department of Biotechnology, Sathyabama Institute of Science and Technology, Chennai 119, Tamil Nadu

2 Grower Life Solutions India Pvt Ltd, Porur, Chennai 116, Tamil Nadu; biologiask@gmail.com (S.K.R.N.); shyamdlmicro@gmail.com (D.S.); m.prabanch@gmail.com (M.M.P.); arvindbharani@gmail.com (R.S.A.B.); govindarajavinash@gmail.com (G.P.A.);

* Correspondence: biologiask@gmail.com;

Scopus Author ID 14023036500

Received: 21.08.2020; Revised: 24.09.2020; Accepted: 26.09.2020; Published: 2.10.2020

\begin{abstract}
Principles of nanoscience and nanotechnology principles has received recent attention in biofilm inhibition strategy. Biofilm - notable microbial growth as cell aggregates on a solid surface is found to be highly susceptible to nanoparticles treatment, which leads to the development of nanoparticles as an effective antimicrobial. In this present study, the anti-biofilm effect of zinc oxide nanoparticles (ZnO NPs) against human pathogenic strains Pseudomonas aeruginosa and Staphylococcus aureus was carried out by microtitre plate spectrophotometric assay. The effect of nanoparticles on the biochemical composition like total carbohydrates (TC), and total protein (TP) of the biofilm matrix was carried out. Biocompatibility or non-target toxic effect was also studied by the determination of cytotoxicity on Vero cells. Results show that the chemical reduction method brought about highly stable nanostructural particles with a size range of 80-90 $\mathrm{nm}$. An anti-biofilm study indicates that the biofilm of both the tested strain was highly susceptible to the nanoparticle treatment as dose-dependent manner. Drastic reduction of total carbohydrates and total protein of the biofilm matrix of tested bacterial strains was observed in nanoparticles treatment. Biocompatibility study with Vero cells reveals that the nanoparticle's treatment was not shown any sign of cytotoxic effect at all the tested dosages, and no sign of apoptosis was observed. The present study implies that the synthesized $\mathrm{ZnO}$ nanoparticles in this present study can be used as an anti-bacterial agent against life-threatening disease-causing organisms.
\end{abstract}

Keywords: ZnO nanoparticles; biofilm; Pseudomonas aeruginosa; Staphylococcus aureus; biofilm matrix; biocompatibility; vero cells.

(C) 2020 by the authors. This article is an open-access article distributed under the terms and conditions of the Creative Commons Attribution (CC BY) license (https://creativecommons.org/licenses/by/4.0/).

\section{Introduction}

Diverse class of substances that have structural components smaller than 100 nanometers (nm) in at least one dimension. Nanomaterials (NMs) include nanoparticles (NPs), which are particles with at least two dimensions between approximately 1 and $100 \mathrm{~nm}$. Have a high surface area to volume ratio, and the number of surface atoms and their arrangement determines the size and properties of the NM. It can be categorized into three types: natural UFPs, incidental NMs, and engineered NMs. Engineered NMs are used in a wide variety of 
applications, including environmental remediation, pollution sensors, photovoltaics, medical imaging, and drug delivery $[1,2]$.

Advantages of nanoparticles are Increased bioavailability, dose proportionality, smaller dose form, increased surface area results in a faster dissolution of the active agent in an aqueous environment, such as the human body. Faster dissolution generally equates greater absorption and bioavailability, and smaller drug doses less toxicity, reduction in fed/fasted variability $[3,4]$.

Among the diverse metallic nanoparticles, zinc oxide nanoparticles have attracted much attention due to their versatile and promising applications in biological sciences, such as an antibacterial, antifungal, and antifouling agent. The safety of zinc nanoparticle and its compatibility with human skin made it a suitable additive for textiles and surfaces that came in contact with the human body [5]. Zinc nanoparticles have been valued in various applications, such as sunscreens, toothpaste, and cosmetics, especially because of their ability to absorb ultraviolet radiation [6,7].

Zinc oxide nanoparticles have been valued in various applications, such as sunscreens, toothpaste, and cosmetics, especially because of their ability to absorb ultraviolet radiation [8]. $\mathrm{ZnO}$ nanoparticles showed bactericidal effects on Gram-positive and Gram-negative bacteria as well as the fungal spores, which were resistant to high temperature and high pressure [9]. An important point about zinc oxide is its opacity. Therefore, in high quantities, it reduces the curing potential of light-cured resin-based composites and deteriorates the mechanical properties. It is important to use this material in small and controlled amounts $[10,11]$. In this study, zinc oxide nanoparticles synthesized by chemical route was checked for the potential anti-biofilm effect against human pathogenic strains followed by the determination of biocompatibility using the Vero cell line as a model system.

\section{Materials and Methods}

\subsection{Synthesis of free $\mathrm{ZnO}$ nanoparticles.}

The synthesis of free $\mathrm{ZnO}$ nanoparticle was carried out by the chemical reduction method. In this method, $0.1 \mathrm{M}$ zinc nitrate was reduced with $0.1 \mathrm{M} \mathrm{NaOH}$ as a dropwise addition under magnetic stirrer. The reaction mixture once turns into white color precipitate, indicates the synthesis of $\mathrm{ZnO}$ nanoparticles. The reaction mixture thus obtained was centrifuged; the collected pellet was lyophilized and used for further studies.

\subsection{Characterization.}

The primary confirmation of synthesized nanoparticles was carried by UV-vis spectroscopy to determine surface plasmon absorbance maxima. UV-vis spectrum analysis was recorded in the Shimadzu-1800 spectrophotometer (800-200nm range). The sample was sonicated prior to the measurement for uniform dispersion. Further characterization of nanoparticles was done by Fourier Transform Infrared Spectroscopy (FTIR) to determine the changes in functional groups. FTIR spectra were measured using Bruker Optic GmbH Tensor 27. The nanoparticles were mixed uniformly with potassium bromide at 1:5 (sample:KBr) ratio, respectively. The $\mathrm{KBr}$ discs were prepared by compressing the powders (mixtures of sample and $\mathrm{KBr}$ ) at a pressure of ton for $5 \mathrm{~min}$ in a hydraulic press. The discs seemed in the range of $400-4000 \mathrm{~cm}^{-1}$. 
Particle shape and size morphology was studied by field emission scanning electron microscopy. In an SEM setup, the nanoparticles coated to be conductive is scanned in a high vacuum chamber with a focussed electron beam using supra 55-carl Zeiss (Germany) magnification range of $35-10.000$, resolution $200 \mathrm{~A}$, acceleration voltage $19 \mathrm{kV}$.

\subsection{Biofilm inhibition study.}

\subsubsection{Bacterial strain and growth condition.}

Bacterial strains used in this study - Pseudomonas aeruginosa and Staphylococcus aureus maintained on tryptic soy agar slant. Tryptic soy broth (Hi media) was used for the inocula preparation of the bacterial strain. Cultures were inoculated from fresh slopes and incubated with shaking at $37^{\circ} \mathrm{C}$ for 24 hours. Cells were collected by centrifugation, and the collected cell debris was washed twice in PBS and suspended to OD520 prior to use in biofilm experiments.

\subsubsection{Nanoparticles induced anti-biofilm effect.}

Nanoparticles induced antibiofilm effect was carried out by 96 wall plates spectrophotometric assay [12]. The known volume of respective bacterial strain inoculum was inoculated into 96 well plates followed by the addition of nanoparticles, incubated $37^{\circ} \mathrm{C}$ for 3 days. After the incubation, the culture was discarded, washed the wells with sterile distilled water. A washed plate was added with $0.1 \mathrm{ml}$ of crystal violet solution for 30 minutes. After the incubation period, the stain was removed, and the wells were washed thoroughly, followed by the addition of $95 \%$ ethanol incubated for 15 minutes. The reaction mixture was read spectrophotometrically at $570 \mathrm{~nm}$. Further confirmation of nanoparticles induced anti biofilms effect was studied by scanning electron microscopy. Adherent biofilm of the respective bacterial strain was processed, and the processed biofilm was examined by SEM Carl Zeisssubra (Germany) scanning electron microscope. Nanoparticles induced effect on the biofilm was also confirmed by the determination of the biochemical composition of the biofilm matrix. Adherent biofilm of the respective bacterial strain was isolated by the sonication method. The biochemical composition of isolated biofilm, mainly total protein and total carbohydrates, was carried out by standard biochemical estimation methods $[13,14]$.

\subsection{Biocompatibility.}

The biocompatibility of synthesized nanoparticles was studied by the determination of cell viability of Vero cells, which was exposed to different concentrations of nanoparticles. Assessment of cell viability using tetrazolium dye MTT assay reveals cell viability [15]. The Vero cell line was obtained from the National center for cell Sciences (NCCS), Pune, India, that maintained on RPMI1640 was used in this assay. Cells were exposed to different concentration of nanoparticles, incubated for $24 \mathrm{hrs}$ at $5 \% \mathrm{CO}_{2}$ incubator washed with phosphate-buffered saline ( $\mathrm{pH} 7.4$ ) followed by addition of $0.5 \%$ 3-(4,5-dimethyl-2-thiazolyl)2,5-diphenyl--tetrazolium bromide (MTT) in phosphate-buffered saline solution. After $4 \mathrm{hrs}$ incubation, $1 \mathrm{ml}$ of DMSO was added. Viable cells were determined by the absorbance at 540 $\mathrm{nm}$. Measurements were performed, and the concentration required for a $50 \%$ inhibition of viability (IC50) was determined. The toxic effect of nanoparticles was also confirmed by the microscopic examination of cells. 


\section{Results and Discussion}

Nano-sized $\mathrm{ZnO}$ displays fluctuating morphologies and shows noteworthy antibacterial action over a wide range of bacterial species investigated by an enormous group of analysts. $\mathrm{ZnO}$ is as of now being explored as an antibacterial specialist in both microscale and nanoscale definitions[16]. $\mathrm{ZnO}$ nanoparticles display noteworthy antimicrobial exercises when molecule size is diminished to the nanometer run; at that point, nano-sized $\mathrm{ZnO}$ can cooperate with the bacterial surface and additionally with the bacterial center where it enters inside the cell, and in this manner, shows unmistakable bactericidal components [17]. In this study, chemically synthesized zinc oxide nanoparticles were evaluated for the anti-biofilm effect against human pathogenic strains. The biocompatibility of the synthesized nanoparticles was also checked on Vero cells.

\subsection{Synthesis and characterization of $\mathrm{ZnO}$ nanoparticles.}

Chemical reduction of zinc nitrate with sodium hydroxide brought about highly stable nanoparticles with high antimicrobial activity. The primary confirmation of synthesis was studied by the conversion of the reaction mixture into white color precipitate. The precipitate thus obtained was centrifuged; the collected pellet was lyophilized and used for further studies.

The optical properties of the ZnO NPs optical properties were studied with the UVvisible absorption spectra in the wavelength range of $200-800 \mathrm{~nm}$. The $\mathrm{ZnO}$ NPs maximum absorption band of UV-visible absorption spectra was found at $207 \mathrm{~nm}$ (Fig 1). This characteristic band can be assigned to the intrinsic band-gap absorption of $\mathrm{ZnO}$ due to the electron transitions from the valence band to the conduction band. A similar observation is reported by Kumar, where UV-vis spectroscopy was used to characterize the optical absorption properties of $\mathrm{ZnO}$. The UV-vis absorption spectra of the samples were recorded at room temperature in the wavelength range of 200 to $800 \mathrm{~nm}$.

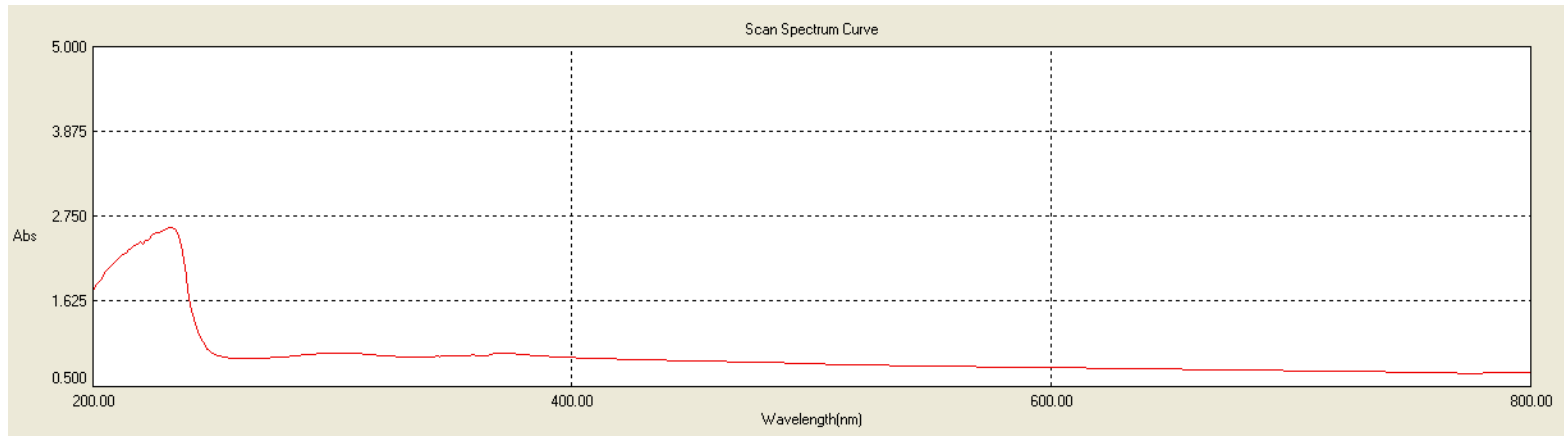

Figure 1. UV visible absorption spectra of $\mathrm{ZnO}$ nanoparticles.

Fourier Transform Infrared Spectroscopy (FTIR) analysis was used to determine the functional groups responsible for the reduction of the precursor (Fig 2).

Nanoparticles were mixed uniformly with potassium bromide at 1:5 (sample: $\mathrm{KBr}$ ) at a pressure of ton for $5 \mathrm{~min}$ in a hydraulic press. The discs seemed in the range of $400-4000 \mathrm{~cm}^{-1}$. Similar findings are recorded where the composition of the sample was analyzed by the FTIR measurement. The absorption band at $1638.01 \mathrm{~cm}^{-1}$ indicates the bending mode of vibration in water $\left(\mathrm{H}_{2} \mathrm{O}\right)$, and the broad absorption band at $3427.50 \mathrm{~cm}^{-1}$ indicates the stretching mode of vibration in the hydroxyl (-OH) group. Particle morphology study was done with scanning electron microscopy (SEM), which shows nanostructural particles with a size range of 80-90 nm. Particles were found to be agglomerated (Fig 3). 


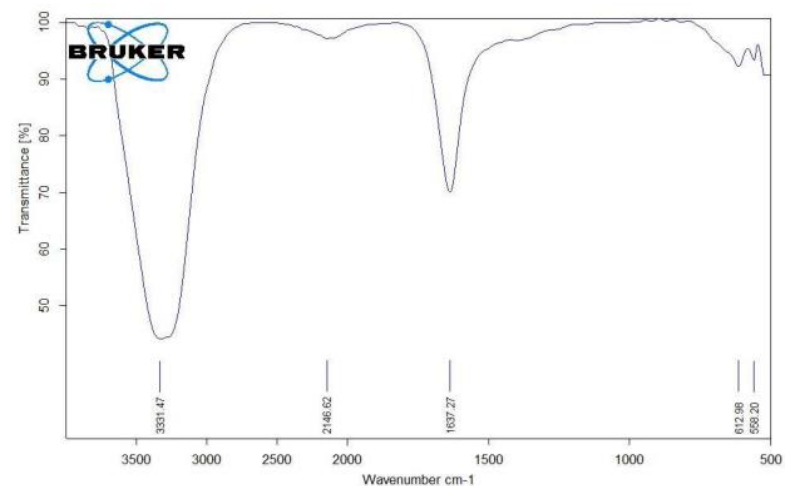

Figure 2. FTIR spectra of $\mathrm{ZnO}$ nanoparticles.

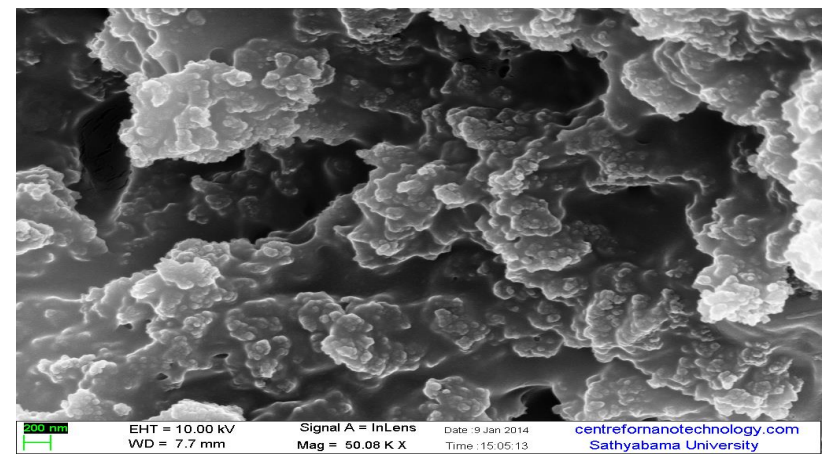

Figure 3. SEM micrograph of $\mathrm{ZnO}$ nanoparticles.

\subsection{Antibiofilm effect.}

Nanoparticles induced anti-biofilm effect was studied by spectrophotometric crystal violet microtitre plate assay against Pseudomonas aeruginosa and Staphylococcus aureus. The inoculum of the respective bacterial strain in the individual wells of the microtitre plate was exposed to different concentrations of nanoparticles treatment. After the incubation period, the developed biofilm was stained, extracted as ethanol solubilized mixture. The determination of the optical density of ethanol solubilized mixture of the respective bacterial strain reveals the biofilm inhibition. Results were represented as inhibition percentage of biofilm development (Fig 4).

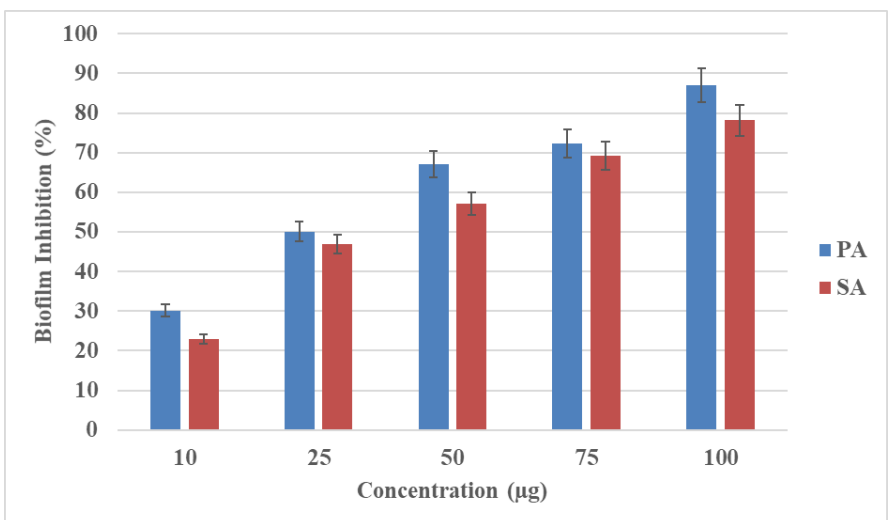

Figure 4. Biofilm inhibition of $\mathrm{ZnO}$ nanoparticles.

Biofilm of both the tested bacterial strains was susceptible to the nanoparticles treatment, and a significant effect was recorded in the high dosage of nanoparticles treatment $(\mathrm{P}=0.05 \%)$. Nanoparticles with $100 \mu \mathrm{g}$ and $75 \mu \mathrm{g}$ exhibited 87.0 and $78.2 \%$ of biofilm inhibition against $P$. aeruginosa and $S$. aureus. Similar findings on nanoparticles induced 
biofilm inhibitory effect against pathogenic bacteria supported our present findings. The synergistic effect of biogenic silver nanoparticles with various plant products and chemotherapeutics against the biofilm of Staphylococcus aureus has been reported [18]. Effect of chemogenic silver nanoparticles coated catheter on biofilm of a clinical isolate of Staphylococcus aureus studied by Namasivayam et al. [19]. Anti biofilm or biofouling effect of metallic nanoparticles against biofilm or biofouling networks of bacterial flora associated with the real water supply of Chennai, Tamil Nadu, India, has recently reported [20]. Scanning electron microscopy analysis indicates that the tested nanoparticles recorded a distinct antibiofilm effect against both the tested strains. Unlike the control groups, the nanoparticles treatment group shows degraded cell masses, which weakened due to nanoparticles treatment (Fig 5).
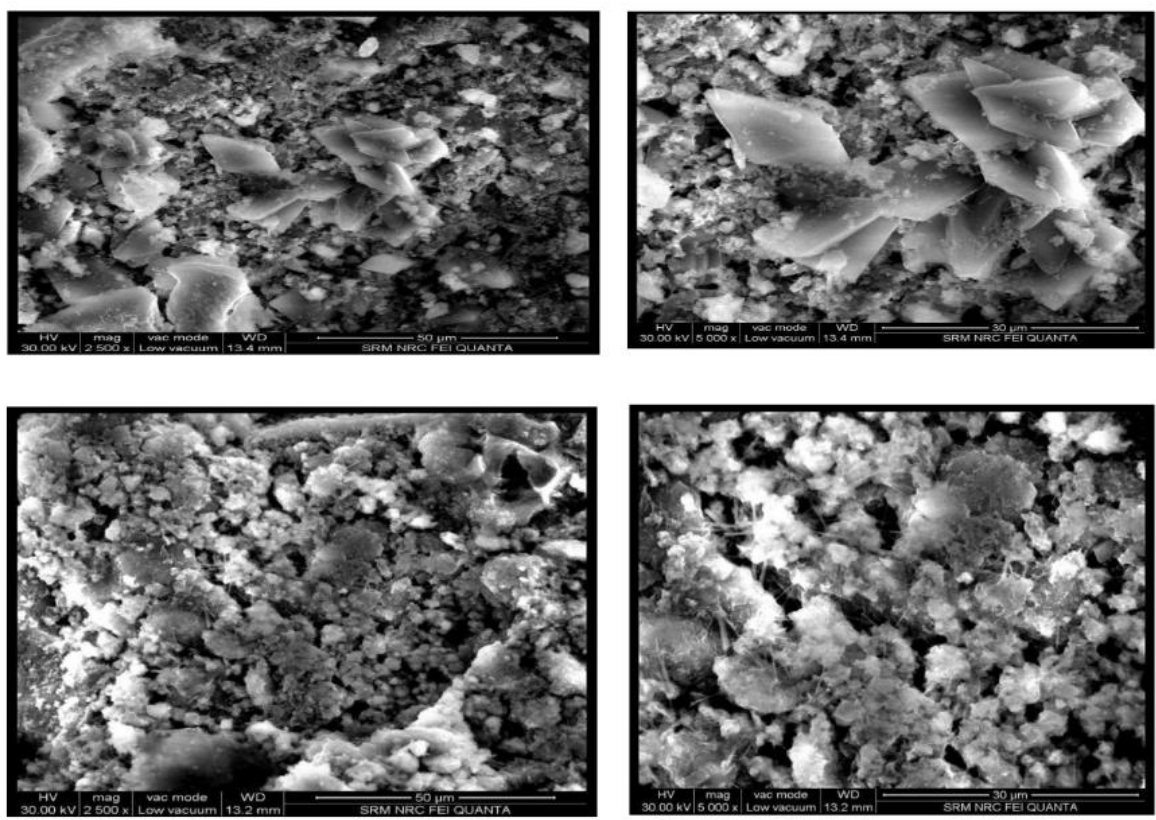

Figure 5. SEM micrograph of biofilm treated with nanoparticles (a) Control-P.aeruginosa (b) control-S.aureus (c) treatment-P.aeruginosa (d) treatment-S.aureus.

Control groups of both the tested bacterial strains show dense cell masses that are bound inside the matrix. The disintegration of biofilm into inactive cell masses in the nanoparticles treatment group confirms the potential anti-biofilm effect of nanoparticles.

The biochemical composition of the biofilm matrix, mainly total carbohydrates (TC) and total protein (TP) was also investigated. Results show that the nanoparticles treatment brought about a drastic reduction of TC and TP (Fig 6).

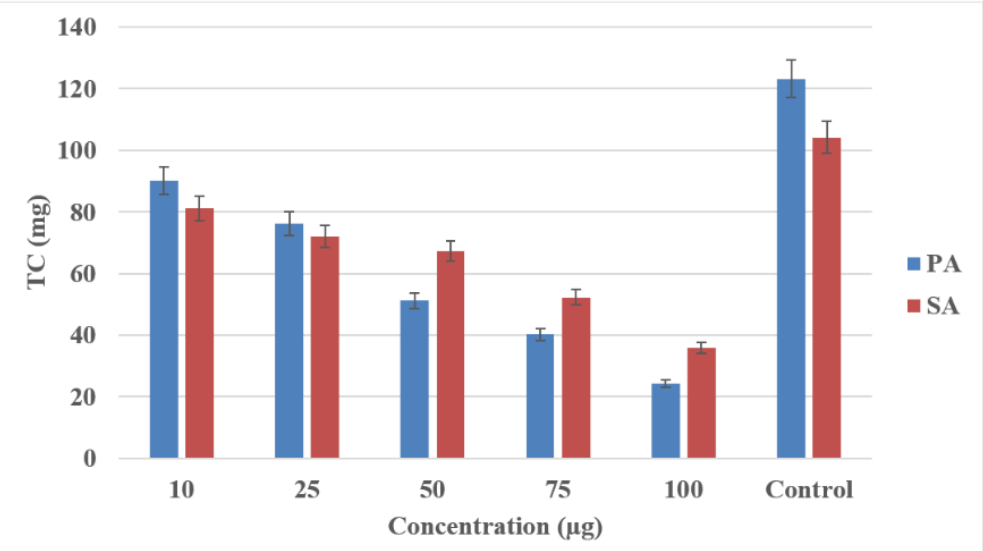

Figure 6. Effect of $\mathrm{ZnO}$ nanoparticles on total carbohydrates of the biofilm matrix. 
Reduction in the biochemical composition of the biofilm matrix due to the nanoparticles treatment may lead to the major reason for the degradation of biofilm[21].

\subsection{Biocompatibility.}

The non-target effect or biocompatibility of synthesized nanoparticles was studied by using the Vero cell line adopting MTT assay. Among the different models, established cell lines play a major role in toxicity assessment. Results indicate that the nanoparticles treatment was not inducing any notable cytotoxic effect on the tested cell line. Nanoparticles with all the dosages except $100 \mu \mathrm{g}$, which shows $74.2 \%$ of viability and the rate of viability was found to increase as the concentration of nanoparticles was decreased (Fig 7).

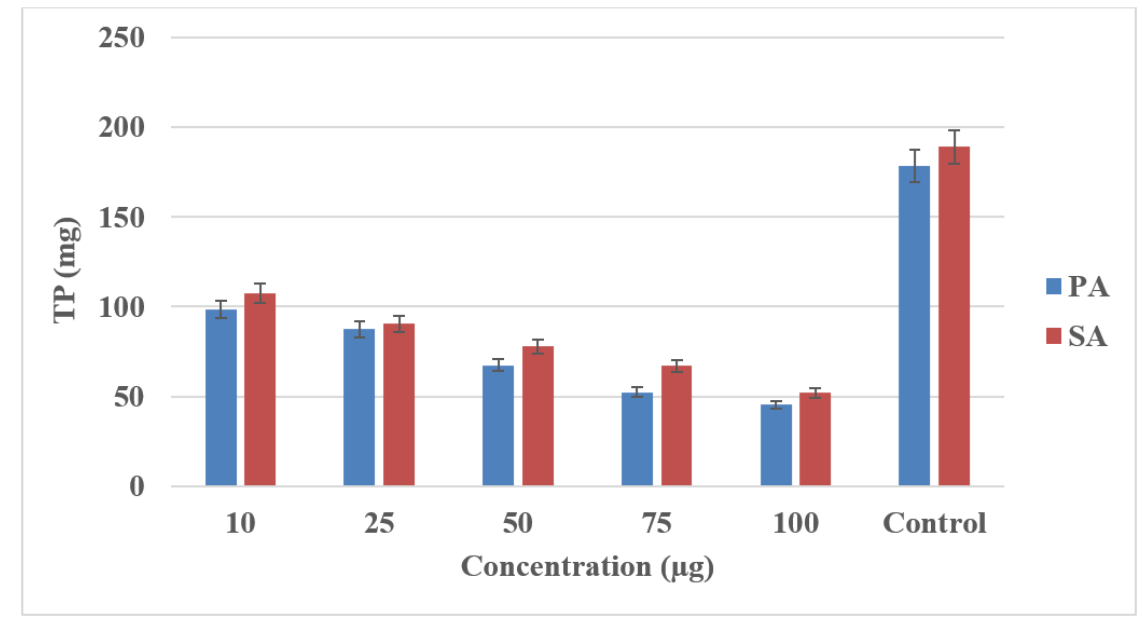

Figure 7. Effect of $\mathrm{ZnO}$ nanoparticles on a total protein of biofilm matrix.

No marked effect on cell viability was recorded in nanoparticles treatment, which reveals the best biocompatibility of nanoparticles. Further confirmation of biocompatibility was done by microscopic examination of Vero cells treated with different dosages. All the tested dosages showed no sign of cytotoxic effect (Fig 8).

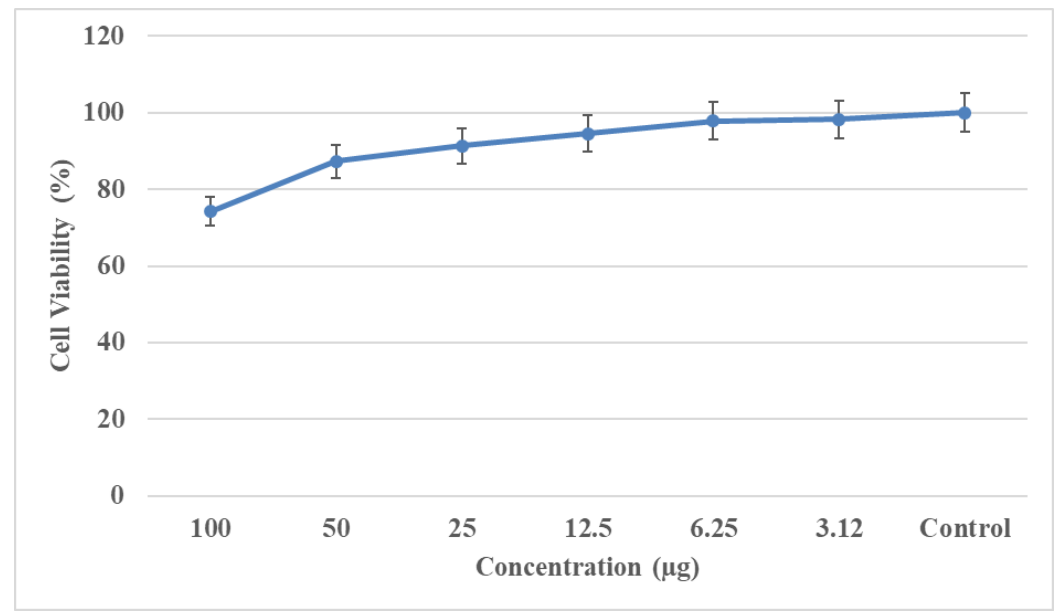

Figure 8. Effect of nanoparticles on cell viability of Vero cells.

It can be clearly observed that the cells treated with nanoparticles with $100 \mu \mathrm{g}$ showed fewer changes in the morphological features, whereas cells treated with the remaining dosages have a well-developed nucleus, devoid of peripheral cellular distribution as in control cells. The cytotoxic effect of nanoparticles on cell viability has a major role in biocompatibility 
assessment, which reveals the tested nanoparticles were not found to interact with functional groups of intracellular proteins, as well as with the nitrogen bases and phosphate groups in DNA. All these findings imply that the potential antibacterial activity and biocompatibility of zinc oxide nanoparticles and this study suggest the possible utilization of $\mathrm{ZnO}$ nanoparticles as an effective antibacterial agent (Fig 9).

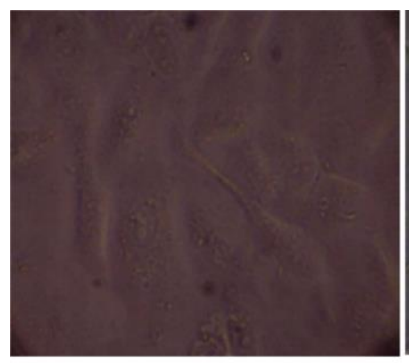

(a)

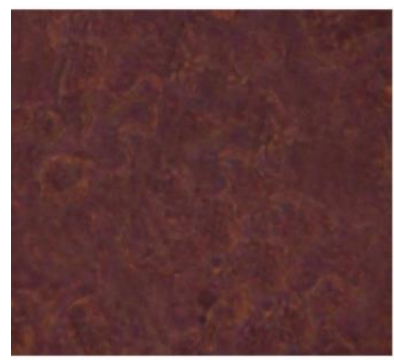

(d)

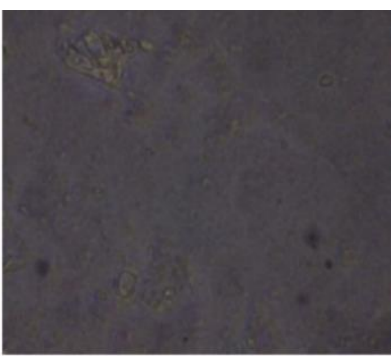

(b)

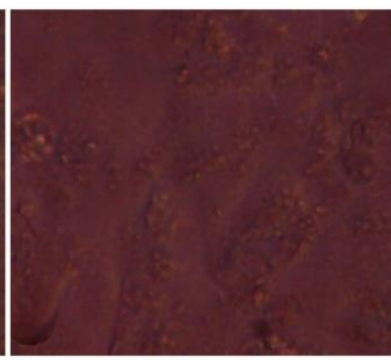

(e)

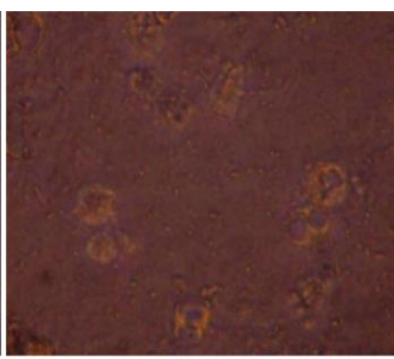

(c)

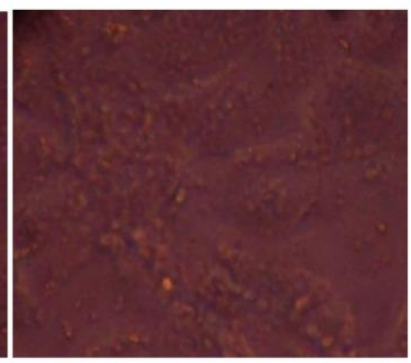

(f)

Figure 9. Microscopic examination of vero cells treated with different concentration of $\mathrm{ZnO}$ nanoparticles (a) control (b) 100 (c) 50 (d)25 (e) 12.5 (f) 6.25 ug of nanoparticles treatment.

\section{Conclusions}

Among the diverse nanomaterials, zinc oxide-based nanoparticles have gained more attention in biomedicine because of the high efficacy and biocompatibility. In this present investigation, highly stable, zinc oxide nanoparticles were synthesized by chemical reduction method with distinct nanoarchitecture. Synthesized nanoparticles recorded potential antibacterial activity by showing anti-biofilm effect against human pathogenic bacterial strains Pseudomonas aeruginosa and Staphylococcus aureus. Nanoparticles induced anti-biofilm effect by showing changes in biofilm architecture and biochemical composition of the biofilm matrix. The present study suggests the possible utilization of nanoparticles as an effective antimicrobial agent against life-threatening infection-causing organisms.

\section{Funding}

This research received no external funding.

\section{Acknowledgments}

Thanks due to IRC, SIST, Chennai, India, for the nanoparticles characterization studies.

\section{Conflicts of Interest}

The authors declare no conflict of interest. 


\section{References}

1. Slavin, Y.N.; Asnis, J.; Häfeli, U.O.; Bach, H. Metal nanoparticles: Understanding the mechanisms behind antibacterial activity. J. Nanobiotechnol. 2015, 15, https://doi.org/10.1186/s12951-017-0308-z.

2. Pang, Z.; Raudonis, R.; Glick, T.J.; Lin, T.J.; Cheng, Z. Antibiotic resistance in Pseudomonas aeruginosa: mechanisms and alternative therapeutic strategies. Biotechnology advances 2015, 15, 177-192, https://doi.org/10.1016/j.biotechadv.2018.11.013.

3. Ali, R.; Shanan, Z.J.; Saleh, G.M.; Abass, Q. Green Synthesis And The Study Of Some Physical Properties Of Mgo Nanoparticles And Their Antibacterial Activity. Iraqi Journal of Science 2020, 61, 266-276, https://doi.org/10.24996/ijs.2020.61.2.9.

4. Tang, Z.X.; Bin-Feng, L.V. MgO Nanoparticles As Antibacterial Agent: Preparation And Activity. Braz J ChemEng.2014, 31, 591-60,. https://doi.org/10.1590/0104-6632.20140313s00002813.

5. Rasmussen, J.W.; Martinez, E.; Louka, P.; Wingett, D.G. Zinc oxide nanoparticles for selective destruction of tumor cells and potential for drug delivery applications. Expert Opin. Drug Deliv. 2010, 7, 1063-1077, https://doi.org/10.1517/17425247.2010.502560.

6. Monopoli, M.P.; Aberg, C.; Salvati, A.; Dawson, K.A. Zinc oxide nanoparticles for selective destruction of tumor cells and potential for drug delivery applications. Nat Nanotechnol. 2012, 7, 779-786, https://doi.org/10.1517/17425247.2010.502560.

7. Zhang, L.; Jiang, Y.; Ding, Y.; Povey, M.; York, D. Investigation into the antibacterial behaviour of suspensions of $\mathrm{ZnO}$ nanoparticles ( $\mathrm{ZnO}$ nanofluids). J NanopartRes. 2007, 9, 479-489, https://doi.org/10.1007/s11051-006-9150-1.

8. Al-Gaashani, R.; Radiman, S.; Al-Douri, Y.; Tabet, N.; Daud, A. R. Investigation of the optical properties of $\mathrm{Mg}(\mathrm{OH})_{2}$ and $\mathrm{MgO}$ nanostructures obtained by microwave-assisted methods. Journal of Alloys and Compounds 2012, 52, 71-76, https://doi.org/10.1016/j.jallcom.2012.01.045.

9. Stankic, S.; Suman, S.; Haque, F.; Vidic, J. Pure and multi metal oxide nanoparticles: synthesis, antibacterial and cytotoxic properties. Journal of Nanobiotechnology 2016, 14, https://doi.org/10.1186/s12951-016-02256.

10. Maji, J.; Pandey, S.; Basu, S. Synthesis and evaluation of antibacterial properties of magnesium oxide nanoparticles. Bulletin of Materials Science 2020, 25, 48-55, https://doi.org/10.1007/s12034-019-1963-5.

11. Dizaj, S.M.; Lotfipour, F.; Barzegar-Jalali, M.; Zarrintan, M.H.; Adibkia, K. Antimicrobial activity of the metals and metal oxide nanoparticles. Mater. Sci. Eng. C. 2014, 44, 278-284, https://doi.org/10.1016/j.msec.2014.08.031.

12. Toole, G.O.; Kaplan, H.B.; Kolter, R. Biofilm formation as microbial development. Annu. Rev. Microbiol. 2000, 54, 49-79, https://doi.org/10.1146/annurev.micro.54.1.49.

13. Dubois, M.; Gilles, K.A.; Hamilton, J.K.; Rebers, P.T.; Smith, F. Colorimetric method for determination of sugars and related substances. Anal. Chem. 1956, 28, 350-356, https://doi.org/10.1038/168167a0.

14. Padmavathy, N.; Vijayaraghavan, R. Enhanced bioactivity of ZnO nanoparticles-an antimicrobial study. SciTechnolAdv Mater. 2008, 9, https://doi.org/10.1088/1468-6996/9/3/035004.

15. Carmichael, J.; DeGraff, W.G.; Gazdar, A.F.; Minna, J.D.; Mitchell, J.B. Evaluation of a Tetrazolium-based Semiautomated Colorimetric Assay: Assessment of Chemosensitivity Testing. Cancer Res.1987, 47, 936942.

16. Singh, R.; Smitha, M.S.; Singh, S.P. The role of nanotechnology in combating multi-drug resistant bacteria. J. Nanosci. Nanotechnol. 2014, 14, 4745-4756, https://doi.org/10.1166/jnn.2014.9527.

17. Mahendra, C.; Manaswini, G.V.; Pavithra, V. A Review on Applications of MgO based Nanocomposite Materials. J. Nanosci. Nanoengg. 2020, 16, 187-194.

18. Namasivayam, S.K.R; Elamathi, K.; Brijesh, K.S. Effect of biologically synthesized silver nanoparticles with plant products and chemotherapeutics against biofilm of clinical isolates of Staphylococcus aureus and Candida tropicalis. J. Biotechnol. Biotherap.2011, 1, 17-21.

19. Namasivayam, S.K.R.; Christo, B.B.; Arasu, S.K.; Kumar, K.A.M.; Deepak, K. Anti biofilm effect of biogenic silver nanoparticles coated medical devices against biofilm of clinical isolate of Staphylococcus aureus. Glob. J. Med. Res. 2013, 13, 1-7.

20. Namasivayam, S.K.R.; Francis, A.L.; Valli, C.N. Bacterial biofilm or biofouling networks with numerous resilience factors from real water supplies of Chennai and their enhanced susceptibility to biocompatible nanoparticles. Journal of Cleaner Production 2019, 231, https://doi.org/10.1016/j.jclepro.2019.05.199.

21. Parsek, M.R.; Greenberg, E.P. Sociomicrobiology: the connections between quorum sensing and biofilms. Journal of Cleaner Production 2005, 13, 27-33, https://doi.org/10.1016/j.tim.2004.11.007. 